\title{
Pluralismo informativo en la era de la deliberación digital: percepciones de periodistas y ciudadanos
}

\section{Informative pluralism in the era of digital deliberation: perceptions of journalists and citizens}

\author{
Juan-Carlos Suárez-Villegas; Ruth Rodríguez-Martínez; Xavier Ramon-Vegas
}

Cómo citar este artículo:

Suárez-Villegas, Juan-Carlos; Rodríguez-Martínez, Ruth; Ramon-Vegas, Xavier (2020). “Pluralismo informativo en la era de la deliberación digital: percepciones de periodistas y ciudadanos". Profesional de la información, v. 29, n. 5, e290525.

https://doi.org/10.3145/epi.2020.sep.25

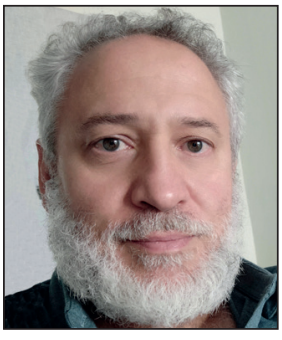

Juan-Carlos Suárez-Villegas https://orcid.org/0000-0002-2199-7028

Universidad de Sevilla

Facultad de Comunicación

Área de Filosofía Moral y Política

Av. Américo Vespucio, $\mathrm{s} / \mathrm{n}$.

41092 Sevilla, España

jcsuarez@us.es

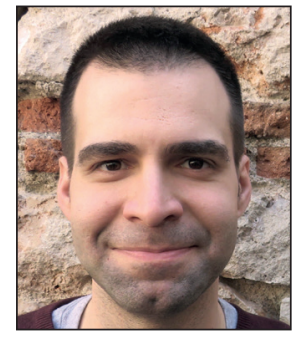

Xavier Ramon-Vegas

https://orcid.org/0000-0002-4478-5626

Universitat Pompeu Fabra

Departament de Comunicació

Roc Boronat, 138

08018 Barcelona, España

xavier.ramon@upf.edu

\section{Resumen}

En un escenario complejo y en profunda transformación, la interacción que implica la comunicación digital ha cambiado de forma irreversible el sentido del pluralismo informativo. En la era de la deliberación digital, los cauces se multiplican y permiten contrastar diversas perspectivas a través de una comunidad de usuarios internacional. Asimismo, las nuevas prácticas participativas en la Web y las redes sociales exigen una mayor transparencia por parte de los medios de comunicación, que ayude a los ciudadanos a tomar decisiones responsables. Empleando metodologías cuantitativas y cualitativas -encuesta a profesionales y seis grupos de discusión con ciudadanos-, esta investigación tiene como propósito conocer la percepción de periodistas y ciudadanos españoles sobre el pluralismo y, concretamente sobre la transparencia y la participación de los usuarios en el ecosistema comunicativo actual. Los resultados señalan que ambos colectivos se muestran favorables a dar a conocer los vínculos institucionales, comerciales y políticos de los medios de comunicación, pero son escépticos respecto a que el entorno digital haya fomentado nuevas formas de transparencia, especialmente entre los medios tradicionales. La opinión de periodistas y ciudadanía se distancia, sin embargo, en un elevado número de cuestiones planteadas. Para los ciudadanos, la participación y la posibilidad de canalizar quejas y sugerencias a través de la web, Twitter y Facebook es positiva y se configura como una práctica cada vez más frecuente. Sin embargo, los periodistas aún muestran una cierta reticencia a interactuar con los lectores y a responder a sus comentarios en las redes sociales.

Financiación

Este artículo forma parte del proyecto de investigación MediaACES, "Accountability y Culturas Periodísticas en España. Impacto y propuesta de buenas prácticas en los medios de comunicación españoles", financiado por el Ministerio de Economía y Competitividad de España y el Fondo Europeo de Desarrollo Regional (Mineco/Feder, UE, ref: CSO2015-66404-P). 


\title{
Palabras clave
}

Pluralismo informativo; Deliberación digital; Participación; Interacción; Transparencia; Vínculos comerciales y políticos; Medios de comunicación; Medios sociales; Facebook, Twitter; Periodistas; Profesionales; Ciudadanos; Audiencias; Usuarios.

\begin{abstract}
In a complex and ever-evolving landscape, the interaction that digital communication provides has irreversibly changed the meaning of media pluralism. In the era of digital deliberation, channels have multiplied, allowing perspectives to be contrasted across an international user community. Likewise, new participatory practices through the web and social networks require greater transparency from media organizations, which helps citizens make responsible decisions. Using quantitative and qualitative methodologies (a survey of professionals and six focus groups with citizens), this research aims to determine the perception of Spanish journalists and citizens on pluralism and, specifically, transparency and user participation in the current communication ecosystem. The results indicate that both groups favor the publicization of the institutional, commercial, and political links of media outlets, but they are skeptical that the digital environment has encouraged new forms of transparency, especially among legacy media. The opinion of journalists and citizens, however, is different on a large number of the issues raised. For citizens, participation and the possibility of channeling complaints and suggestions through the web, Twitter, and Facebook is considered to be positive and to represent an increasingly frequent practice. However, journalists still show a certain reluctance to interact with readers and respond to their comments on social networks.
\end{abstract}

\section{Keywords}

Media pluralism; Digital deliberation; Participation; Interaction; Transparency; Commercial and political links; Media; Social media; Facebook; Twitter; Journalists; Professionals; Citizens; Audiences; Users.

\section{Introducción}

A diferencia de la posición pasiva que presuponían los medios de comunicación tradicionales, el ecosistema actual de comunicación digital concede a la audiencia un papel activo como creador de contenidos o prod-user, tal y como los denomina Bruns (2008). A esta actividad cabría añadir también la participación de la audiencia en los sitios web de los medios de comunicación y en las cuentas oficiales de los medios en redes sociales como Twitter y Facebook (Bruns, 2015; Bruns; Highfield, 2012; Ruiz-Caballero et al., 2010; 2011). Los comentarios en las webs y las redes sociales ofrecen nuevas posibilidades para promover debates espontáneos pero organizados sobre cuestiones de interés público. Estos espacios virtuales de interacción ayudan a fortalecer el espíritu crítico y conectan a la ciudadanía con todos los ámbitos de la sociedad, incluida la política (Fenoll; Cano-Orón, 2017; Muñiz et al., 2019; Valera-Ordaz, 2017; 2019).

La interacción que implica la comunicación digital ha cambiado de forma irreversible el sentido del pluralismo informativo (Valcke; Sükösd; Picard, 2015). En la era de la deliberación digital (Masip; Ruiz-Caballero; Suau, 2019; Raeijmaekers; Maeseele, 2015) los cauces se multiplican y permiten contrastar diversas perspectivas a través de una comunidad de usuarios internacional. De acuerdo con Habermas, la esfera pública es un espacio donde los ciudadanos

"pueden juntarse y unirse libremente y expresar y publicar sus opiniones libremente" (Habermas, 2006, p. 103).

En la esfera pública digital, los comentarios a través de las webs y las redes sociales son una oportunidad de primer orden para fomentar la deliberación y trascender las limitaciones de los mecanismos de participación clásicos como las cartas al director. Tradicionalmente, las cuatro reglas que han marcado la selección de dichas cartas -relevancia, entretenimiento, brevedad y autoridad- han limitado de forma clara la diversidad de voces así como la publicación de críticas hacia los propios medios de comunicación (Torres-da-Silva, 2012; Wahl-Jorgensen, 2002).

Los comentarios en las webs y las redes sociales permiten así incrementar el pluralismo espontáneo de una ciudadanía activa, creando espacios de interacción que enriquecen el debate público, lo que Castells (2009) califica como "auto-comunicación de masas". Se configura así un modelo de comunicación multinodal entre los distintos agentes, un "periodismo ambiental" en el que interviene el estado de conciencia de un público activo hacia los acontecimientos sociales (Hermida, 2010).

Dicho esto, los avances tecnológicos no han revertido necesariamente en una mayor calidad de las contribuciones de los usuarios, puesto que no siempre se cumplen las condiciones óptimas para la deliberación: universalismo, inclusividad, racionalidad y civilidad (Jacobs; Cook; Delli-Carpini, 2009). Como señalan Ruiz-Caballero et al.,

"Ios nuevos espacios propiciados por la tecnología se han convertido en avenidas por donde transitan inmensos caudales de opinión que posibilitan la impunidad desde la participación muchas veces anónima” (Ruiz-Caballero et al. (2010, p. 10).

Existen casos de quienes acuden a dichos foros con una actitud disruptiva, para quienes se ha acuñado el término de trol (Eberwein, 2019; Fenoll, 2015). Los comentarios son un espacio donde pueden aflorar las descalificaciones, provocaciones e incluso el lenguaje del odio hacia determinados grupos sociales (Santana, 2015). Estos problemas han Ilevado a varias agencias y medios de referencia internacionales -como NPR, CNN o Reuters- a eliminar los comentarios y blogs, restringiendo las posibilidades de participación a las redes sociales (Liu; McLeod, 2019). 
La polarización de las conversaciones es otro rasgo frecuente en estos espacios, particularmente en determinadas culturas periodísticas. En su análisis de más de 15.000 comentarios en cinco periódicos digitales de referencia de varios países, Ruiz-Caballero et al. (2011, p. 482) señalaron que en los países pertenecientes al modelo mediterráneo o pluralista polarizado (Hallin; Mancini, 2004) -entre ellos España- se mostraba

"un menor respeto entre los participantes y un menor pluralismo".

En un escenario marcado por la problemática expansión de la desinformación (Allcott; Gentzkow; Yu, 2019; Wardle; Derakhshan, 2017) y la creciente polarización política y social (Suhay; Bello-Pardo; Maurer, 2018), la polarización de las conversaciones puede contribuir al "encogimiento del pluralismo" (Ruiz-Caballero et al., 2010, p. 33). Pese a estas carencias, los comentarios aportan beneficios democráticos, tales como

"la autoexpresión ciudadana, la socialización democrática y el refuerzo de la cohesión social" (Valera-Ordaz, 2017, p. 1).

En la actualidad, la producción y el consumo de noticias, marcados por los algoritmos de los motores de búsqueda y la creciente dependencia de las métricas (Ferrucci, 2020; Nieborg; Poell, 2018), privilegian la práctica del clickbait para atraer la atención de los lectores, soslayando de este modo la diversidad, pluralidad y calidad de los contenidos (Dweyer, 2019; Dweyer; Martin, 2017; Parcu, 2019). Con las debidas cautelas exigidas para validar su contenido con rigor profesional, los medios también pueden aprovechar las aportaciones realizadas por los usuarios a través de los comentarios y otros espacios de participación para dar voz a aquellos sectores o colectivos que tienen menos representación en la sociedad. En su ejercicio, el periodista puede acotar estas aportaciones y presentarlas como contenido informativo, facilitando una comprensión más completa de los acontecimientos y problemas sociales. De este modo, las aplicaciones de participación pueden ayudar a los medios a cumplir con su cometido normativo de fomentar la diversidad de voces, cuestiones y perspectivas en los espacios informativos (Aznar et al., 2018; Steiner; Magin; Stark, 2019).

Aunque los periodistas no siempre se han mostrado favorables a las nuevas formas de participación (Hermida; Thurman, 2008; Ruiz-Caballero et al., 2010), cada vez son más conscientes de los comentarios realizados por los usuarios y responden a ellos (Fengler et al., 2015). Pese a todo, los resultados del proyecto Media Accountability and Transparency in Europe (MediaAcT), en el que se encuestó a profesionales de 14 países (Alemania, Austria, España, Estonia, Finlandia, Francia, Holanda, Italia, Jordania, Polonia, Reino Unido, Rumanía, Suiza y Túnez), atestiguan que los profesionales son aún reticentes a afirmar que las críticas realizadas por los usuarios a través de los comentarios afectan de forma significativa a su práctica profesional (Fengler et al., 2015). En la actualidad,

“muchas redacciones muestran una fatiga participativa en lugar de una cultura participativa” (Porlezza, 2019, p. 2).

Por otro lado, investigaciones recientes con ciudadanos demuestran la importancia que este colectivo otorga a las posibilidades de participación. Mediante la realización de 22 entrevistas en profundidad a ciudadanos en Alemania, Austria y Suiza, Eberwein (2019) reveló que los usuarios consideran los comentarios como las formas de participación más valiosas. Según este estudio, los motivos por los cuales participan a través de los comentarios son la búsqueda de la verdad, la formación de opinión, la provocación, el entretenimiento o el deseo de canalizar sus críticas y descontento respecto a la calidad de los contenidos periodísticos.

En la misma línea, a través de la realización de 26 entrevistas semiestructuradas con usuarios alemanes, Engelke destacó que la audiencia

“percibe y valora la naturaleza y el potencial deliberativo de las secciones de comentarios" (Engelke, 2019, p. 462).

Este trabajo también señaló que

"la audiencia quiere que los periodistas retengan un cierto poder en la fase de interpretación del proceso informativo a través de la moderación de los comentarios, la participación en los debates y estableciendo umbrales más elevados" (Engelke, 2019, p. 462).

Así pues, los ciudadanos perciben que la responsabilidad en los espacios de deliberación digital debe ser compartida entre los profesionales y los usuarios.

En un sentido amplio, el pluralismo también exige fomentar la transparencia. Como señala el Media Pluralism Monitor Report 2017 (Masip; Ruiz-Caballero; Suau, 2018), en el escenario español nos encontramos en un contexto caracterizado por la agudización de problemas clásicos en la profesión que inciden directamente sobre la independencia informativa: las injerencias políticas e interferencias comerciales (Berganza; Van-Dalen; Chaparro-Domínguez, 2010; Masip, 2018). Estos problemas son indisociables de las características de los sistemas de medios pertenecientes al modelo pluralista polarizado (Hallin; Mancini, 2004), entre las que cabe destacar

- elevada politización y polarización de la vida pública;

- influencia de la clase política sobre los medios;

- profesionalización débil;

- limitada autonomía periodística.

Teniendo en cuenta la situación actual y estos condicionantes, la transparencia cobra una importancia de primer orden. La transparencia puede definirse como la 
“apertura de información por parte de las instituciones públicas y de otras entidades sin restricciones, de forma que los datos que se aporten sean fiables y actualizados para que la ciudadanía pueda tomar decisiones responsables" (Díez-Garrido; Campos-Domínguez; Calvo, 2019, p. 85).

España se ha caracterizado por una escasa cultura de transparencia, hecho que explica la tardía introducción de legislación en esta materia (Díez-Garrido; Campos-Domínguez; Calvo, 2019). La presente Ley 19/2013, de 9 de diciembre, de transparencia, acceso a la información pública y buen gobierno, tiene tres objetivos fundamentales:

"garantizar el derecho de todos los ciudadanos a acceder a la información, hacer cumplir la transparencia de todas las administraciones públicas, y supervisar la observación de prácticas de gobernanza justas" (Appelgren; Salaverría, 2018, p. 988).

Según el estudio realizado por Appelgren y Salaverría (2018), los periodistas españoles valoran positivamente la introducción de esta legislación, pero manifiestan las dificultades aún existentes para acceder a la información pública, entre ellas la organización de los documentos y el tiempo que transcurre entre la realización de una petición de acceso y su aprobación por parte de las autoridades. Mediante una encuesta realizada a periodistas españoles, Díez-Garrido y Campos-Domínguez (2018) señalaron que los profesionales consideran que las medidas aportadas por esta ley son insuficientes. Los periodistas encuestados valoraron de forma negativa el nivel de transparencia exhibido por las instituciones españolas. Estos resultados ponen de manifiesto que los periodistas demandan una mayor cultura de transparencia a los poderes públicos. Aun así, es un reto pendiente examinar de qué forma valoran el nivel de transparencia que debe exigirse a los propios medios de comunicación.

En el ámbito periodístico, la transparencia puede entenderse como la difusión pública de información relevante sobre la estructura, organización, propiedad, situación financiera y vínculos económicos y políticos de los medios de comunicación (Heikkilä et al., 2012). También se refiere a la responsabilidad de los medios de explicar los procesos editoriales y las prácticas profesionales que llevan a cabo (Diakopoulos; Koliska, 2017). Por último, la transparencia también requiere revelar

"las relaciones de interactividad que el medio mantiene con su público" (Campos-Domínguez; Redondo-García, 2015, p. 195).

La transparencia puede fomentarse a través de varios instrumentos de rendición de cuentas que

“permiten a la audiencia valorar mejor la calidad de un proceso o producto periodístico" (Fengler et al., 2014, p. 21).

Entre estas herramientas de accountability, cabe destacar:

- los blogs editoriales (Ramon-Vegas; Rojas-Torrijos; Billings, 2019; Spiller et al., 2016);

- las páginas de transparencia corporativa en las webs de los medios (Campos-Domínguez; Redondo-García, 2015);

- otros mecanismos de verificación, rectificación y réplica, como por ejemplo los botones de notificación de errores (Karlsson; Clerwall; Nord, 2017; Mauri-Ríos; Ramon-Vegas, 2015; Zeng; Burgess; Bruns, 2019).

En su análisis de seis medios de comunicación públicos y privados de Italia, Portugal y España (RAI, Repubblica, RTP, Público, TVE y El país), Campos-Domínguez y Redondo-García (2015) destacaron que el nivel de transparencia mediática era incipiente, tanto en las fuentes de financiación como en la explicación de los criterios editoriales. Las autoras también señalaron la opacidad de dichos medios respecto a

"los criterios de participación y compromiso del medio con la respuesta a contenidos elaborados por los ciudadanos" (Campos-Domínguez; Redondo-García, 2015, p. 203).

En una investigación reciente sobre cuatro medios digitales españoles (Eldiario.es, Elconfidencial.com, Elpais.com y Elmundo.es), Pérez-Díaz, Zamora-Medina y Arroyas-Langa (2020) señalaron que dichos medios han incorporado mecanismos de transparencia, aunque únicamente Eldiario.es exhibe un mayor compromiso por revelar sus fuentes de financiación.

Según los resultados del proyecto MediaAcT, mencionado anteriormente, los periodistas europeos sostienen que

"es importante ser transparente sobre la misión, los códigos éticos, la información sobre la propiedad y sobre los intereses políticos y empresariales" (Groenhart; Evers, 2014, p. 142).

De acuerdo con el estudio de Eberwein (2019), los usuarios también desean un mayor nivel de transparencia sobre la propiedad y orientación de los medios, sobre los flujos de trabajo editoriales y sobre los procesos de toma de decisiones en el ámbito periodístico.

\section{Metodología}

Esta investigación tiene como objetivo conocer la opinión de periodistas y ciudadanos españoles sobre el pluralismo informativo, concretamente sobre la transparencia y la participación de los usuarios. La siguiente pregunta ha guiado la investigación:

PI: ¿De qué forma los periodistas y ciudadanos españoles valoran la transparencia y la participación de los usuarios en el proceso informativo? 
Para la consecución del propósito se han planteado los siguientes objetivos de investigación:

OI1: Determinar cómo los periodistas y ciudadanos españoles valoran diversos aspectos relacionados con el pluralismo informativo.

Ol2: Conocer la opinión de los periodistas y ciudadanos españoles sobre la participación de los usuarios en la web del medio de comunicación, incluyendo el derecho de expresar quejas sobre el tratamiento informativo y sugerir correcciones.

Ol3: Conocer la opinión de los periodistas y ciudadanos españoles sobre la participación de los usuarios en las cuentas de Facebook y Twitter del medio de comunicación.

Para llevar a cabo la presente investigación se triangularon dos tipos de metodologías (Brennen, 2017):

- en primer lugar, se empleó una metodología cuantitativa a través de un cuestionario online que permitió conocer la opinión de los periodistas sobre diversos aspectos de la profesión periodística, el pluralismo informativo y, concretamente sobre la participación e interacción de los usuarios en las distintas fases del proceso informativo;

- la segunda metodología utilizada fue cualitativa: para conocer la opinión de los ciudadanos sobre diversos aspectos de la profesión periodística y, en concreto, sobre el concepto de pluralidad y el papel de la participación de la audiencia en el proceso informativo, se empleó la técnica de los grupos de discusión.

Se diseñó un cuestionario de 29 preguntas en el que, con el fin de garantizar su operatividad, se combinaron preguntas dicotómicas, de opción múltiple y en forma de escala de valoración (Wimmer; Dominick, 2011). También se permitió a los informantes anotar de forma libre otros comentarios y observaciones relevantes sobre las cuestiones tratadas. Si bien el cuestionario estaba formado por 29 preguntas, en el presente trabajo se han seleccionado aquellas que están estrechamente relacionadas con las condiciones que garantizan un pluralismo informativo efectivo (tablas 1-3). En ellas se pidió a los periodistas que valoraran supuestos del ejercicio periodístico, el pluralismo informativo y la participación e interacción de los usuarios. Los profesionales consultados debían responder en una escala de 0 a 10 , en la que el valor 0 equivalía a "Nada importante" y el valor 10 significaba "Muy importante" para mostrar su conformidad o no con los supuestos.

Tabla 1. Valore la importancia de los siguientes supuestos vinculados con el pluralismo informativo

\begin{tabular}{|l|}
\hline Los medios de comunicación deben dar a conocer sus vínculos comerciales o políticos (si los tienen) \\
\hline Los medios de comunicación deben dar a conocer sus vínculos institucionales y los subsidios y ayudas públicas que recibe \\
\hline Los medios de comunicación deben publicar su cuenta de resultados y detallar de dónde provienen sus ingresos \\
\hline El entorno digital fomenta nuevas formas de transparencia y control de la calidad informativa \\
\hline Los periodistas tienen en cuenta e incorporan las críticas que reciben del público \\
\hline Proporcionar un modo de contacto visible y accesible para remitir quejas sobre el contenido publicado \\
\hline
\end{tabular}

Tabla 2. Valore la importancia de los siguientes supuestos sobre la participación de los usuarios en la web del medio de comunicación

\begin{tabular}{|l|}
\hline Tener en consideración los comentarios de los usuarios en las noticias publicadas en la web del medio \\
\hline Tener en cuenta las correcciones a las noticias publicadas en la web del medio \\
\hline Contestar a los comentarios de las noticias de las que usted es autor \\
\hline Hacer accesible el contacto de los lectores con los redactores de su medio \\
\hline La existencia de una sección con contenido creado por los usuarios \\
\hline Facilitar que los usuarios sugieran noticias a los redactores \\
\hline
\end{tabular}

Tabla 3. Valore la importancia de los siguientes supuestos sobre la interacción de los usuarios a través de las redes sociales

\begin{tabular}{|l|}
\hline Hay que tener en consideración los comentarios de las noticias compartidas en la página Facebook oficial del medio \\
\hline Hay que tener en consideración los comentarios de las noticias compartidas en la cuenta Twitter oficial del medio \\
\hline Hay que contestar a los comentarios de las noticias de Facebook en el caso que usted sea el autor \\
\hline Hay que contestar a los comentarios de las noticias de Twitter en el caso que usted sea el autor \\
\hline
\end{tabular}

Siguiendo los criterios señalados por Weischenberg, Malik y Scholl (2006, p. 227), los encuestados debían reunir las siguientes características:

- trabajar para un medio de comunicación periodística -quedaban por tanto excluidos los profesionales que desempeñasen tareas de relaciones públicas-;

- realizar el ejercicio periodístico, de manera que quedaban excluidos los profesionales que desempeñasen tareas técnicas o de organización en la industria de los medios de comunicación;

- tener una ocupación a tiempo completo o principalmente, es decir, ganar el 50 por ciento o más de sus ingresos de su profesión como periodista. En este caso, los freelances se incluían si ganaban 50 por ciento o más de sus ingresos con actividades periodísticas. 
A diferencia de otros países europeos como Alemania, Francia, Finlandia o Suiza, España no cuenta con un censo o directorio oficial de los profesionales del periodismo (Rodríguez-Martínez; Mauri-Ríos; Fedele, 2017). Con el objetivo de obtener un número suficiente de respuestas en la encuesta, se buscó la colaboración entre varias instituciones profesionales -Federación de Asociaciones de Periodistas de España (FAPE), Colegio de Periodistas de Cataluña, y Asociación de la Prensa de Madrid (APM)-, que distribuyeron el cuestionario entre sus afiliados. El cuestionario se administró de forma online a través de la plataforma SurveyMonkey, estando abierta durante tres meses (17 de octubre de 2017 - 17 de enero de 2018).

Durante el período en el que estuvo activa la encuesta se fueron monitorizando semanalmente las respuestas introducidas. El total de respuestas obtenidas fue de 228 ( $N=228)$. Un 52,2\% ( $n=119)$ de los informantes fueron mujeres y un $47,8 \%$ ( $n=109)$ fueron hombres. La mayoría de los encuestados $(71,1 \%)$ contaba con formación universitaria sobre periodismo. Según su ocupación profesional por tipo de medio:

- $29 \%$ de los periodistas trabajaban para emisoras de radio;

- 26\% para cadenas de televisión;

- 24,1\% para medios impresos (periódicos, publicaciones semanales o revistas);

- 9,2\% para medios online;

- 9,2\% para agencias informativas;

- 7,9\% eran autónomos (freelance).

Una vez recogido el material, se llevó a cabo el análisis estadístico empleando el software especializado IBM Statistical Package for the Social Sciences (SPSS).

Los datos resultantes se triangularon posteriormente con la información cualitativa obtenida de los focus groups con ciudadanos. Se llevaron a cabo 6 grupos focales en las siguientes ciudades españolas:

- Barcelona (GD-01)

- Castellón (GD-02)

- Madrid (GD-03)

- Sevilla (GD-04)

- Mondragón (GD-05)

- Santiago de Compostela (GD-06).

Uno de los aspectos que se tuvo en cuenta fue que el período de realización de los focus groups no superara el mes y medio para que el contexto mediático fuera lo más parecido posible, por lo que el primer focus group que se realizó en Barcelona tuvo lugar el 12 de abril de 2018 y el último en Santiago de Compostela el 31 de mayo de ese mismo año.

Para los seis grupos focales se contó con la participación de 38 personas en total, 22 mujeres y 16 hombres. Para obtener la muestra de participantes se empleó la estrategia de reclutamiento dirigida por investigadores o research-driven recruitment strategy (Peek; Fothergill, 2009): los miembros del equipo de investigación -compuesto por 6 universidades con sede en las ciudades mencionadas- fueron responsables de reclutar a los participantes de cada grupo focal. En el proceso de selección de la muestra, se atendió tanto al criterio de homogeneidad o afinidad temática (todos los participantes debían ser consumidores de información periodística a través de una o varias plataformas) como al criterio de heterogeneidad, que se garantiza con la diversidad de características sociodemográficas (Bryman, 2016). La edad de los participantes está equilibrada, con un $42 \%$ situado en edades medias y el resto repartido casi a partes iguales entre los menores de 30 años y los mayores de 60. La mayoría de las personas acceden a la información mediante los periódicos digitales y las redes sociales (Twitter y Facebook). Además de periódicos generalistas a nivel nacional, como El país, Público, Eldiario.es, El mundo o El confidencial, los participantes mencionaron consumir periódicos regionales, como La voz de Galicia, Levante, VilaWeb, Berria o El diario vasco. En general, los participantes se mostraron interesados por obtener información de diferentes medios y plataformas.

El guion de los grupos de discusión, basado en el cuestionario de la encuesta a periodistas, se centró en temas del ejercicio periodístico, el pluralismo informativo y, concretamente, sobre la transparencia y la participación e interacción de los usuarios en el proceso informativo. Disponer de una guía flexible permitió orientar de una forma efectiva el desarrollo de los grupos de discusión (Tracy, 2020), posibilitando también que emergieran otras dimensiones a partir de la interacción espontánea entre los participantes. Las seis sesiones fueron moderadas por dos profesionales de una empresa especializada, que condujeron el desarrollo de los grupos con la supervisión de miembros del equipo de investigación. Cada una de las sesiones fue grabada en audio y transcrita de forma íntegra para su posterior análisis. Con el objetivo de garantizar la confidencialidad en la presentación de los datos, las citas se presentan con el identificador del focus group y el género de cada participante (H: Hombre; M: Mujer).

\section{Resultados}

\subsection{Percepción de los periodistas sobre el pluralismo informativo}

Cuando se pregunta a los periodistas sobre la importancia que conceden a diversos aspectos vinculados con el pluralismo informativo, uno de los supuestos que consideran fundamental es la posibilidad de proporcionar un modo de contac- 
to visible y accesible a los usuarios para remitir quejas sobre el contenido publicado. Este es con diferencia el supuesto que mayor aceptación ha recibido entre los periodistas consultados, ya que ha obtenido un 9 de media (tabla 4). Resulta interesante este dato ya que, como se verá más adelante, los ciudadanos corroboran esta necesidad de que se puedan canalizar quejas sobre el contenido publicado. Este resultado contrasta con los dos supuestos menos respaldados por los periodistas consultados:

- necesidad de que los periodistas tengan en cuenta e incorporen las críticas que reciben del público, que obtiene la media más baja con un 5,41;

- la idea de que el entorno digital ha fomentado nuevas formas de transparencia y control de la calidad informativa, valorado con un 5,50 .

Esto permite observar un cierto escepticismo por parte de los periodistas consultados sobre los posibles aspectos positivos que ha supuesto la consolidación de internet.

Vinculados asimismo con la idea de transparencia están dos de los supuestos que mejor valoración obtienen entre los periodistas: necesidad de dar a conocer los vínculos institucionales o subsidios y ayudas públicas que reciben (valorado con un 8,66), y los vínculos comerciales y políticos (valorado con un 8,35). Esta necesidad de informar a los usuarios de los vínculos de los medios, en el caso de que existan, es uno de los aspectos a los que los ciudadanos consultados conceden mayor importancia, tal y como se verá más adelante.

Tabla 4. Valoración de los periodistas sobre supuestos de pluralismo informativo

\begin{tabular}{|c|c|c|}
\hline Supuestos & $\begin{array}{l}\text { Puntuación } \\
\text { media }\end{array}$ & $\begin{array}{l}\text { Desviación } \\
\text { típica }\end{array}$ \\
\hline Los medios de comunicación deben dar a conocer sus vínculos comerciales o políticos (si los tienen) & 8,35 & 2,17 \\
\hline $\begin{array}{l}\text { Los medios de comunicación deben dar a conocer sus vínculos institucionales y los subsidios y ayudas } \\
\text { públicas que reciben }\end{array}$ & 8,66 & 1,97 \\
\hline $\begin{array}{l}\text { Los medios de comunicación deben publicar su cuenta de resultados y detallar de dónde provienen sus } \\
\text { ingresos }\end{array}$ & 7,79 & 2,71 \\
\hline El entorno digital fomenta nuevas formas de transparencia y control de la calidad informativa & 5,50 & 3,09 \\
\hline Los periodistas tienen en cuenta e incorporan las críticas que reciben del público & 5,41 & 2,26 \\
\hline Proporcionar un modo de contacto visible y accesible para remitir quejas sobre el contenido publicado & 9,00 & 1,37 \\
\hline
\end{tabular}

Cuando se pregunta a los periodistas sobre la participación de los usuarios es preciso destacar en primer lugar que ninguno de los supuestos alcanza valores superiores a 6,50. El supuesto que mayor aceptación tiene entre los periodistas consultados es la opción de "Tener en cuenta las correcciones a las noticias publicadas en la web del medio", que alcanza un 6,19 de media (tabla 5). El resto no alcanza el 6 de media, y recibe la puntuación más baja, con un 3,58, la "Existencia de una sección con contenido creado por los usuarios". El escaso entusiasmo de los periodistas consultados sobre la participación de los usuarios contrasta, como se verá más adelante, con las respuestas de los ciudadanos, quienes consideran que la participación ha sido clave en la transformación que han vivido los medios de comunicación en los últimos años.

El escaso entusiasmo de los periodistas sobre la participación de los usuarios contrasta con las respuestas de los ciudadanos, quienes consideran que la participación ha sido clave en la transformación de los medios

Tabla 5. Valoración de los periodistas sobre la participación de los usuarios en la web del medio de comunicación

\begin{tabular}{|c|c|c|}
\hline Supuestos & $\begin{array}{c}\text { Puntuación } \\
\text { media }\end{array}$ & $\begin{array}{c}\text { Desviación } \\
\text { típica }\end{array}$ \\
\hline Tener en consideración los comentarios de los usuarios en las noticias publicadas en la web del medio & 5,38 & 2,50 \\
\hline Tener en consideración los comentarios que hacen en las redes sociales & 5,11 & 2,50 \\
\hline Tener en cuenta las correcciones a las noticias publicadas en la web del medio & 6,19 & 2,41 \\
\hline Contestar a los comentarios de las noticias de las que usted es autor & 5,44 & 3,07 \\
\hline Hacer accesible el contacto de los lectores con los redactores de su medio & 5,75 & 2,72 \\
\hline La existencia de una sección con contenido creado por los usuarios & 3,58 & 2,80 \\
\hline Facilitar que los usuarios sugieran noticias a los redactores & 5,51 & 2,82 \\
\hline
\end{tabular}

Cuando se pregunta a los periodistas a propósito de la interacción de los usuarios en las páginas oficiales que el medio tiene en Facebook y Twitter, los resultados apenas muestran diferencias entre ambas redes sociales, pero sí destaca la baja aceptación que reciben por parte de los periodistas (tabla 6). Así por ejemplo, la pregunta de si "Hay que tener en consideración los comentarios de las noticias compartidas en la página de Facebook del medio" obtiene un 5,29, mientras que la equivalente para Twitter consigue un 5,27. Los supuestos que preguntan a los periodistas sobre la necesidad 
de contestar a los comentarios que hacen los usuarios a noticias publicadas en Facebook o Twitter tampoco ofrece diferencias destacables entre una red social u otra. Lo que sí resulta relevante es que ninguna de ellas alcanza el cinco de media, ya que la opción de contestar los comentarios a las noticias en Facebook consigue un 4,75 y las publicadas en Twitter un 4,70.

Tabla 6. Valoración de los periodistas sobre la interacción de los usuarios a través de las redes sociales

\begin{tabular}{|c|c|c|}
\hline Supuestos & $\begin{array}{c}\text { Pun- } \\
\text { tuación } \\
\text { media }\end{array}$ & $\begin{array}{l}\text { Desvia- } \\
\text { ción típica }\end{array}$ \\
\hline Hay que tener en consideración los comentarios de las noticias compartidas en la página Facebook oficial del medio & 5,29 & 2,51 \\
\hline Hay que tener en consideración los comentarios de las noticias compartidas en la cuenta Twitter oficial del medio & 5,27 & 2,54 \\
\hline Hay que contestar a los comentarios de las noticias de Facebook en el caso que usted sea el autor & 4,75 & 3,03 \\
\hline Hay que contestar a los comentarios de las noticias de Twitter en el caso que usted sea el autor & 4,70 & 2,98 \\
\hline
\end{tabular}

\subsection{Percepción de los ciudadanos sobre el pluralismo informativo}

En los focus groups realizados se pusieron de manifiesto seis condiciones fundamentales que los medios de comunicación debían cumplir para garantizar una información plural.

\section{Honestidad a la hora de explicar las posiciones ideológicas del medio y permitir que los periodistas opinen sin presio- nes ideológicas}

De acuerdo con las opiniones expresadas por los ciudadanos consultados, es necesario establecer criterios de calidad que garanticen el pluralismo informativo. Por ejemplo, es preciso diferenciar entre la perspectiva ideológica del medio, que es legítima dado que establece un criterio o punto de vista en un contexto de pluralidad democrática, y la influencia directa de un partido político sobre el medio, que se percibe como un acto publicitario y una dependencia que no permite asumir una visión crítica.

“Es que es muy difícil, porque es normal que un periodista, y que en un periódico en sí haya una línea ideológica. Ahora, que esta línea ideológica sea inmediatamente un partido político, o sea... no sé cómo explicarte... deberían desvincularse de los partidos políticos, y no con la ideología, con lo cual tú sigues proporcionando una información que es normal que tenga tu punto de vista, pero no encaje totalmente en una línea de partido, porque ya... si no, es propaganda totalmente (GD04-M).

\section{Independencia económica}

Los ciudadanos consultados comparten la percepción de que existe mayor libertad para informar y opinar en los medios de comunicación asociados a internet y las redes sociales. Se considera que estos periódicos online tienen menos estructuras y menor dependencia de financiación que, por ejemplo, la prensa escrita tradicional. Los medios digitales pueden por tanto asumir un mayor nivel de libertad para comunicar y aportan "oxígeno" al contexto mediático actual, ya que se perciben como más independientes y aportan mayor pluralidad al abanico de medios y voces diversas.

"Creo que internet, y lo digo también por experiencia como socia en eldiario.es, ha permitido que aparezca una prensa, que no voy a ser tan ingenua para pensar que no es totalmente libre, pero tiene menos ataduras económicas que otras, porque el mantenimiento financiero de esas estructuras es más sencillo. Entonces no es lo mismo poner una rotativa en marcha que da trabajo a doscientas personas y pagar las luces y los edificios que la libertad que da que estemos un grupo pequeño de personas, en una estructura pequeña. Creo que esto es un oxígeno para una prensa distinta" (GD01-M).

Asimismo, sugieren ejemplos hipotéticos de empresas que podrían influir en el tratamiento informativo de ciertas informaciones, pero también mencionan casos reales. Este es el caso, según comentan algunos participantes, de la financiación proveniente de entidades financieras a medios de comunicación o el capital israelí con el que cuenta el diario El país (Adar Capital Partners).

"Por ejemplo, El país, una de sus fuentes de ingresos es un fondo de capital israelí, entonces eso se nota mucho porque está muy sesgado cuando dan la información de lo que está ocurriendo ahora en Gaza. Es muy difícil que lo veamos en la primera plana y ¿por qué?, porque un fondo que financia a El país... Eso la gente no lo sabe. Si los lectores supieran que parte de la financiación de El país viene de un fondo de capital israelí, a lo mejor eso explica el tratamiento que están dando a lo que está ocurriendo ahora en Gaza. Entonces como lectores, claro que tenemos el derecho de saberlo y no nos lo dicen. Para mí es fundamental” (GD03-M).

"Eldiario.es justo sacaron la cuenta de resultados y ahí decían que ellos no tenían ninguna hipoteca, o sea ningún crédito con ningún banco, y claro eso los hace más independientes. Todos los demás medios sí que tienen acuerdos de financiación con bancos. Lógicamente, estaría muy bien saber todo eso porque al final es eso lo que les está atando, y eso es lo que está haciendo que la independencia no exista, es lo que hace que estén los periodistas con las manos atadas" (GD03-M). 


\section{Transparencia}

Para la mayoría de los participantes de los focus groups el pluralismo guarda una estrecha relación con la transparencia, lo que implica que los medios deben publicar su cuenta de resultados y detallar de dónde provienen sus ingresos. La publicación de esta información genera aprobación general por parte de los ciudadanos consultados, especialmente en el contexto de desconfianza actual que impera entre la audiencia.

"Yo creo que sí que tiene que ver porque el periodismo no es solamente cómo se enfocan las noticias, es que hay un trasfondo que si de verdad saliera todo a la luz... No me imagino el mapa que saldría con unas relaciones entre otros, yo que sé empresas petrolíferas por decir algo ¿no? Yo sí que creo que estaría bien saberlo, ahora bien, me parece una utopía pensar que eso se puede llegar a desenredar algo" (GD02-M).

\section{Derecho del público a expresar sus quejas por el tratamiento informativo y recibir feedback por parte de los perio-} distas

Los ciudadanos consultados creen que los medios de pequeña estructura son por lo general más accesibles y canalizan mejor las quejas que llegan desde el público. Este mecanismo es considerado fundamental para ayudar a los profesionales del periodismo a hacer mejor su trabajo y ser más conscientes de la importancia de la audiencia. Algunos de los participantes plantean que estas quejas sirvan a los medios para reflexionar sobre cómo mejorar las noticias.

"Igual se debería hacer, independientemente de si reciben cartas o no, una autocrítica de lo que se ha hecho cada x tiempo, o sea que se pueda repasar qué se está haciendo y se puede deliberar de alguna manera dentro de la institución, qué no se hace" (GD01-M).

Resulta por ello fundamental que la participación de la audiencia no sea retórica, sino que sea considerada como un recurso informativo de interés público. Los ciudadanos consultados consideran imprescindible que se tengan en cuenta y se respondan los comentarios de los usuarios en las cuentas oficiales de Facebook y Twitter de los medios.

\section{Es fundamental que la participación de la audiencia no sea retórica, sino que sea considerada como un recurso informati- vo de interés público}

“Esto está creciendo a base exponencial. Eso creo que [los medios] lo tienen muy claro" (GD01-H).

Cuando se pregunta a los ciudadanos sobre la necesidad de responder las quejas de la audiencia en las redes sociales, estos responden que si la queja ha generado interés entre la audiencia o se ha replicado en las redes, el medio de comunicación debe garantizar esta respuesta.

"A veces hay gente que critica una noticia por haberse adelantado por ejemplo y ya enseguida hay medios que piden disculpas, o sea es un hecho muy pequeño, pero sí que es verdad que se ve ya en Twitter cómo hay comunicaciones con muchos medios" (GD01-H).

"Sí, pero hasta que no se ven perjudicados por que el número de personas que se han quejado es muy grande, no van a reaccionar porque ellos siguen su línea. Si una persona les dice: oye, que te has pasado aquí; pues ah, bueno; pero si es mucha gente [...] entonces sí que luego piden perdón porque les interesa mantener una buena imagen para atraer a lectores" (GD01-M).

"Yo creo que al final se responderá casi automáticamente a cualquier persona, habrá gente encargada que lo decida" (GD01-H).

\section{Participación del público en la producción y corrección de las noticias}

La participación del público en la producción de noticias es considerada por varios de los ciudadanos consultados como uno de los aspectos que define el pluralismo informativo actual. Uno de los participantes denomina este tipo de periodismo como "periodismo ciudadano" y lo valora de forma muy positiva. De hecho, algunos de los ciudadanos destacan que estas aportaciones son más veraces que las de los propios periodistas.

"Pienso que la participación de la audiencia es lo que está haciendo que haya este cambio, o sea hay un cambio en los medios de comunicación y es a través de fact check que hacen los lectores, los usuarios, las personas que están conectadas a internet, y es gracias a ellos muchas veces, como se generan noticias bien o al menos se sigue el hilo conductor para investigar una cosa que te están diciendo: oye, esto está pasando y no lo estás dando o esto lo estás dando y está mal, es un bulo. Entonces al final pienso que la participación de la audiencia está haciendo que el periodismo ciudadano que está habiendo ahora y que está teniendo tanto boom nos venga por una parte bien y por una parte nos venga mal. La parte mala es cuando no se hace crítica de lo que te están comentando o de lo que estás recibiendo por parte de esa audiencia, pero nos viene bien también para decir: uy, es interesante esto que me están diciendo, vamos a buscar y a lo mejor esto llega a esta parte; es el caso de Cristina Cifuentes con el máster" (GD03-M).

Sin embargo, algunos participantes advierten de que este tipo de contenidos recogen puntos de vista parciales que no aportan veracidad a la información. En este sentido, se indica la necesidad de que los medios hagan una selección previa 
de estas opiniones para garantizar una visión plural de la realidad. Es por ello, que la contribución de la audiencia en la corrección de las noticias genera dudas entre los participantes de los grupos focales.

"Yo creo que la corrección de las noticias, a ver... para algo hay profesionales que se han pasado mucho tiempo estudiando, para ser periodistas [...] en la corrección de las noticias creo que tiene que haber profesionales" (GD01-M).

"Me parece irrelevante. Es decir, si el asesinato se cometió a las 12 o a la 1, no cambia el hecho de que haya habido un asesinato... Lo que importa es la estructura" (GD04-H).

Evitar ser un mero altavoz de las instituciones y empresas y editar las noticias con criterios informativos

Cuando se pregunta sobre la falta de elaboración y verificación por parte de los medios de las noticias que llegan de agencias $u$ otras instituciones, los ciudadanos consultados rechazan que los medios repliquen y publiquen las noticias de forma casi idéntica. Añaden asimismo que este tipo de tratamiento informativo pone de manifiesto la ausencia de recursos económicos de los medios de comunicación actuales.

“¿Como se nutren de información? Pues de información de agencias, después de información que viene del grupo al cual pertenecen y después otra cosa más [...] Como ciudadano me parece grave que por ejemplo las páginas de información comarcal se nutran de las notas de prensa que vienen de las instituciones. No tienen periodistas en las redacciones. La información institucional que llega de un ayuntamiento o de una empresa lo que hacen es cortar y pegar. ¿Por qué? Pues entre otras cosas porque no tienen personal para reelaborar esa información, porque no tienen tiempo tampoco para contrastarlas y que no sea lo que dicta la institución, que no se limite a una única fuente. Un partido o una empresa siempre es un punto de vista muy interesado" (GD02-H).

"Entonces eso repercute en que los lectores no saben todo este entramado que hay aquí detrás y dan por válidas cosas que leen que en realidad vienen directamente de la empresa, y eso es un flaquísimo favor, para mí es la gran lacra" (GD03-M).

Algunos participantes afirman que los acontecimientos deben ir más allá de los partidos políticos y el enfoque de las informaciones debe centrarse en los hechos, no tanto en las opiniones o posicionamiento de los partidos

La labor de contraste de los periodistas es considerada un factor necesario y fundamental políticos. Esta idea contradice en cambio la opinión de otros ciudadanos para quienes las opiniones de los actores políticos son relevantes. Ahora bien, en estos casos la labor de contraste de los periodistas es considerada un factor necesario y fundamental.

"Yo me estoy preguntando si hay que hablar de los políticos o hay que hablar de los acontecimientos sociales y económicos [...] ¿Por qué hay que hablar de los partidos?" (GD05-H).

"Vale y, ¿qué opina el PP, qué opina Podemos, que opina H, que opina...? Tú ves la televisión y escuchas la opinión del PNV porqué ha hecho esto, pero ¿qué opinión tiene el resto sobre lo mismo? En muchos casos hay acontecimientos diarios, te dan la noticia según el partido, y después ya no hay ni un contraste" (GD05-H).

\section{Discusión y conclusiones}

En un escenario complejo y en profunda transformación, el pluralismo informativo se configura como pilar esencial para luchar contra la crisis de confianza y credibilidad de los medios y asegurar una esfera pública vital y democrática (Hanitzsch; Van Dalen; Steindl, 2018; Valcke; Sükösd; Picard, 2015). Uno de los aspectos que propone este trabajo es conocer y comparar la perspectiva de los periodistas y los ciudadanos sobre cuestiones relacionadas con el pluralismo informativo. Si bien la participación ciudadana ha jugado un papel fundamental en los cambios que han experimentado los medios de comunicación en los últimos años, son escasas las investigaciones que tienen en cuenta la perspectiva de los usuarios en relación con las cuestiones analizadas en la presente propuesta.

Cuando se pregunta a periodistas y ciudadanos si los medios digitales cuentan con mecanismos para favorecer el pluralismo informativo y la transparencia, ambos colectivos consideran que estos mecanismos existen, pero señalan la dificultad de que estos valores sean puestos en práctica. Por ejemplo, los periodistas valoran de forma muy positiva la necesidad de dar a conocer los vínculos institucionales o subsidios y ayudas públicas que reciben $(8,66)$, así como los vínculos comerciales y políticos en el caso de que existan $(8,35)$. Pero cuando se les pregunta si el entorno digital ha ayudado de forma real a fomentar nuevas formas de transparencia y control de la calidad informativa las respuestas apenas superan el cinco de media $(5,50)$. Esta visión escéptica coincide con la opinión de los ciudadanos que, por un lado, aseguran que los medios deberían explicar sus ataduras económicas y políticas y, por otro, reconocen que muchos de ellos mantienen intereses políticos y económicos de los que los lectores no son conscientes. Aunque es cierto que algunos participantes consultados sí valoran la aparición de nuevos medios digitales que, a diferencia de los tradicionales, tienen menos ataduras comerciales y explican su situación financiera con mayor transparencia.

De forma coherente con investigaciones precedentes (Eberwein, 2019; Groenhart; Evers, 2014), los resultados del estudio confirman que la transparencia se configura, desde el punto de vista profesional y ciudadano, como un valor fun- 
damental para salvaguardar el pluralismo informativo (OI1). Teniendo en cuenta ambas perspectivas, los medios de comunicación deben ahondar en el compromiso con la transparencia desarrollado desde múltiples ámbitos (Appelgren; Salaverría, 2018; Díez-Garrido; Campos-Domínguez; Calvo, 2019) para fomentar su propia transparencia organizativa, editorial y participativa (Diakopoulos; Koliska, 2017; Heikkilä et al. 2012; Campos-Domínguez; Redondo-García, 2015). Exhibir una mayor transparencia mediática puede ayudar a cultivar un mayor conocimiento entre los ciudadanos de la actividad periodística, la calidad de los contenidos y de los condicionantes que afectan la práctica profesional en el contexto español, entre los que cabe destacar la polarización, la precarización y las injerencias de índole política y económica (Campos-Domínguez; Redondo-García, 2015; Hallin; Mancini, 2004; Masip; Ruiz-Caballero; Suau, 2018).

La opinión de periodistas y ciudadanía se distancia, sin embargo, en un elevado número de cuestiones planteadas. Por ejemplo, cuando se pregunta a los periodistas sobre la necesidad de canalizar quejas sobre el contenido publicado, estos se muestran reticentes y se obtiene un 5,41 de media. Para los ciudadanos en cambio, la posibilidad de que los usuarios hagan llegar las quejas a los medios de comunicación resulta fundamental, pero admiten que la mayor parte de los medios responden y atienden las quejas de los lectores e, incluso piden disculpas, porque les interesa mantener una buena imagen para atraer a lectores. También resulta notorio el escaso respaldo que los supuestos sobre la participación de los usuarios reciben entre los periodistas, lo que contrasta de forma clara con el parecer de los ciudadanos, para quienes la participación de los usuarios resulta primordial y es, sin duda, el elemento que más ha contribuido a la transformación de los medios de comunicación (OI2). Las actitudes respecto a la participación observadas en el caso español respaldan los resultados de investigaciones precedentes en varios contextos periodísticos, que apuntaban al escepticismo por parte de los profesionales (Fengler et al., 2015; Hermida; Thurman, 2008; Porlezza, 2019) y a la valoración positiva de los nuevos espacios de deliberación digital por parte de los ciudadanos (Eberwein, 2019; Engelke, 2019).

De los siete supuestos consultados a los periodistas sobre la participación de los usuarios, la media más alta se sitúa en el 6,16, cuando los periodistas muestran su opinión sobre la necesidad de tener en cuenta las correcciones a las noticias publicadas en la web del medio. Este es el supuesto que, en cambio, genera dudas entre los ciudadanos consultados, ya que consideran que esto debe recaer en los profesionales del periodismo. Si se presta atención al resto de preguntas se observa que ninguna alcanza el 6 de media y la puntuación más baja la obtiene "La existencia de una sección con contenido creado por los usuarios" con un 3,58.

Una distancia similar entre periodistas y ciudadanos se aprecia cuando se pregunta a ambos sobre la necesidad de que el periodista tenga en cuenta o responda a los comentarios publicados en las páginas oficiales que el medio tiene en Facebook o Twitter. Como se ha señalado previamente, las redes sociales se han configurado como nuevos espacios de deliberación que permiten superar las limitaciones de mecanismos tradicionales, pese a generar también nuevos retos participativos (Eberwein, 2019; Fenoll, 2015; Ruiz-Caballero et al., 2011; Valera-Ordaz, 2019). Las cifras obtenidas por los cuatro supuestos sobre estas cuestiones oscilan entre el 5,29 y el 4,70, poniendo de manifiesto la escasa importancia que otorgan los periodistas a la interacción de los usuarios a través de estas dos redes sociales. Esto contrasta de forma clara con la percepción de los ciudadanos, para quienes la interacción a través de las redes sociales es positiva y se configura como una práctica cada vez más frecuente en el actual ecosistema comunicativo (OI3). Sin embargo, ambas partes señalan que este desiderátum no siempre se ve reflejado en la práctica efectiva de los medios, que no disponen aún de vías suficientes para visibilizar los efectos de dicha interacción con el público.

Debido al valor periodístico y comercial que aportan estos nuevos canales (Liu; McLeod, 2019; Ruiz-Caballero et al., 2010), los medios de comunicación deben trabajar para aplicar varios mecanismos para monitorizar la participación y salvaguardar la calidad de las conversaciones, tanto en sus sitios web como en sus perfiles en las redes sociales. Entre estos mecanismos, cabe destacar el establecimiento de condiciones de registro más estrictas, la creación de normas de participación que guíen las contribuciones de los usuarios y una mayor intervención periodística en forma de moderación de los comentarios (Engelke, 2019; Mauri-Ríos; Ramon-Vegas, 2015; Suhay; Bello-Pardo; Maurer, 2018; Ruiz-Caballero et al., 2011). Paralelamente, los medios deben educar a los usuarios en los beneficios de una participación constructiva y que contribuya a aportar puntos de vista que completen y enriquezcan la tarea periodística.

Partiendo de los resultados obtenidos en el presente trabajo, futuras investigaciones deberán seguir ahondando en el análisis de la participación y la transparencia como indicadores clave del pluralismo mediático. Nuevos estudios deberán monitorizar de qué forma los espacios y aplicaciones que fomentan la participación y transparencia evolucionan en medios de diferente titularidad y tipo. Mediante la aplicación del análisis del contenido, se pueden examinar los sitios web de medios de comunicación, facilitando la realización de análisis comparativos a nivel nacional e internacional. Siguiendo la línea emprendida en el presente trabajo, investigaciones futuras deberán seguir evaluando de qué forma los periodistas y ciudadanos valoran las condiciones que permiten el desarrollo de un pluralismo efectivo. La combinación de técnicas cuantitativas y cualitativas (encuestas, grupos de discusión y entrevistas) permitirá seguir arrojando luz sobre esta cuestión fundamental para la salud democrática en general y el sistema mediático en particular. 


\section{Referencias}

Allcott, Hunt; Gentzkow, Matthew; Yu, Chuan (2019). "Trends in the diffusion of misinformation on social media". Research \& politics.

https://doi.org/10.1177/2053168019848554

Appelgren, Ester; Salaverría, Ramón (2018). “The promise of the transparency culture: A comparative study of access to public data in Spanish and Swedish newsrooms". Journalism practice, v. 12, n. 8, pp. 986-996.

https://doi.org/10.1080/17512786.2018.1511823

Aznar, Hugo; Pérez-Gabaldón, Marta; Alonso, Elvira; Edo, Aurora (eds.) (2018). El derecho de acceso a los medios de comunicación. Valencia: Tirant lo blanch. ISBN: 9788417508449

Berganza, María-Rosa; Van-Dalen, Arjen; Chaparro-Domínguez, María-Ángeles (2010). “La percepción mutua de las relaciones entre periodistas parlamentarios y miembros del congreso y de su influencia en las agendas política y mediática". Revista de comunicación, n. 9, pp. 7-25.

https://revistadecomunicacion.com/pdf/2010/Art007-25.pdf

Brennen, Bonnie S. (2017). Qualitative research methods for media studies. New York: Routledge. ISBN: 9781315435978

Bruns, Axel (2008). Blogs, Wikipedia, Second Life and beyond: from production to produsage. New York: Peter Lang. ISBN: 9780820488660

Bruns, Axel (2015). "Making sense of society through social media”. Social media + society, v. 1, n. 1. https://doi.org/10.1177/2056305115578679

Bruns, Axel; Highfield, Tim (2012). "Blogs, Twitter, and breaking news: The produsage of citizen journalism”. In: Lind, Rebecca-Ann (ed.). Produsing theory in a digital world: The intersection of audiences and production. New York: Peter Lang, pp. 15-32. ISBN: 9781433115196

https://eprints.qut.edu.au/53339

Bryman, Alan (2016). Social research methods. Oxford: Oxford University Press. ISBN: 9780199689453

Campos-Domínguez, Eva; Redondo-García, Marta (2015). “Meta periodismo y transparencia informativa en el periodismo del siglo XXI". Obets. Revista de ciencias sociales, v. 10, n. 1, pp. 185-209.

https://doi.org/10.14198/OBETS2015.10.1.07

Castells, Manuel (2009). Comunicación y poder. Madrid: Alianza editorial. ISBN: 9788420684994

Diakopoulos, Nicholas; Koliska, Michael (2017). "Algorithmic transparency in the news media". Digital journalism, v. 5, n. 7, pp. 809-828.

https://doi.org/10.1080/21670811.2016.1208053

Díez-Garrido, María; Campos-Domínguez, Eva (2018). “Los periodistas españoles ante la transparencia en España: Valoración y uso de la apertura informativa". Revista española de la transparencia, n. 7, pp. 49-69.

http://uvadoc.uva.es/handle/10324/42107

Díez-Garrido, María; Campos-Domínguez, Eva; Calvo, Dafne (2019). “La transparencia de los partidos políticos como estrategia electoral. Una evaluación de sus promesas y sus páginas web". Trípodos, n. 44, pp. 83-104.

http://www.tripodos.com/index.php/Facultat_Comunicacio_Blanquerna/article/view/620

Dweyer, Tim (2019). “Media pluralism policies and the implications of social news sharing”. In: Martin, Fiona; Dwyer, Tim (eds.). Sharing news online. Commendary cultures and social media news ecologies. Cham, Suiza: Palgrave Macmillan, pp. 223-256. ISBN: 9783030179052

https://doi.org/10.1007/978-3-030-17906-9_8

Dweyer, Tim; Martin, Fiona (2017). "Sharing news online. Social media news analytics and their implications for media pluralism policies". Digital journalism, v. 5, n. 8, pp. 1080-1100.

https://doi.org/10.1080/21670811.2017.1338527

Eberwein, Tobias (2019). "'Trolls' or 'warriors of faith'? Differentiating dysfunctional forms of media criticism in online comments". Journal of information, communication and ethics in society, v. ahead-of-print.

https://doi.org/10.1108/JICES-08-2019-0090

Engelke, Katherine M. (2019). “Enriching the conversation: audience perspectives on the deliberative nature and potential of user comments for news media". Digital journalism, v. 8, n. 4, pp. 447-466.

https://doi.org/10.1080/21670811.2019.1680567 
Fengler, Susanne; Eberwein, Tobias; Leppik-Bork, Tanja; Lönnendonker, Julia; Pies, Judith (2014). “Introduction: media accountability - Basic concepts and theoretical foundations". In: Fengler, Susanne; Eberwein, Tobias; Mazzoleni, Gianpietro; Porlezza, Colin (eds). Journalists and media accountability. An international study of news people in the digital age. New York: Peter lang, pp. 7-30. ISBN: 9781433122811

https://doi.org/10.3726/978-1-4539-1247-8

Fengler, Susanne; Eberwein, Tobias; Alsius, Salvador; Baisnée, Olivier; Bichler, Klaus; Dobek-Ostrowska, Boguslawa; Evers, Huub; Glowacki, Michal; Groenhart, Harmen; Harro-Loit, Halliki; Heikkilä, Heikki; Jempson, Mike; Karmasin, Matthias; Lauk, Epp; Lönnendonker, Julia; Mauri-Ríos, Marcel; Mazzoleni, Gianpietro; Pies, Judith; Porlezza, Colin; Powell, Wayne; Radu, Raluca; Rodríguez-Martínez, Ruth; Russ-Mohl, Stephan; Schneider-Mombaur, Laura; Splendore, Sergio; Väliverronen, Jari; Vera-Zambrano, Sandra (2015). "How effective is media self-regulation? Results from a comparative survey on European journalists". European journal of communication, v. 30, n. 3, pp. 249-266.

https://doi.org/10.1177/0267323114561009

Fenoll, Vicente (2015). "El trol de internet. Estrategias de los usuarios para controlar el diálogo en los medios digitales durante el juicio de Francisco Camps". Revista Dígitos, n.1, pp.179-197.

https://revistadigitos.com/index.php/digitos/article/view/9

Fenoll, Vicente; Cano-Orón, Lorena (2017). “Citizen engagement on Spanish political parties' Facebook pages: Analysis of the 2015 electoral campaign comments". Communication \& society, v. 30, n. 4, pp. 131-147.

https://revistas.unav.edu/index.php/communication-and-society/article/view/35763

https://doi.org/10.15581/003.30.4.131-145

Ferrucci, Patrick (2020). "It is in the numbers: how market orientation impacts journalists' use of news metrics". Journalism, v. 21, n. 2, pp. 244-261.

https://doi.org/10.1177/1464884918807056

Groenhart, Harmen; Evers, Hubb (2014). "Media accountability and transparency - What newsrooms (could) do". In: Fengler, Susanne; Eberwein, Tobias; Mazzoleni, Gianpietro; Porlezza, Colin (eds). Journalists and media accountability. An international study of news people in the digital age. New York: Peter lang, pp. 129-145. ISBN: 9781433122811 https://doi.org/10.3726/978-1-4539-1247-8

Habermas, Jürgen (2006). "Political communication in media society: Does democracy still enjoy an epistemic dimension? The impact of normative theory on empirical research". Communication theory, v. 16, n. 4, pp. 411-426.

https://doi.org/10.1111/j.1468-2885.2006.00280.x

Hallin, Daniel C.; Mancini, Paolo (2004). Comparing media systems: three models of media and politics. Cambridge: Cambridge University Press. ISBN: 9780511790867

Hanitzsch, Thomas; Van-Dalen, Arjen; Steindl, Nina (2018). "Caught in the nexus: A comparative and longitudinal analysis of public trust in the press". The international journal of press/politics, v. 23, n. 1, pp. 3-23.

https://doi.org/10.1177/1940161217740695

Heikkilä, Heikki; Domingo, David; Pies, Judith; Głowacki, Michal; Kuś, Michal; Baisnée, Olivier (2012). Media accountability goes online: a transnational study on emerging practices and innovations. Tampere: Journalism Research and Development Centre.

http://www.mediaact.eu/fileadmin/user_upload/WP4_Outcomes/WP4_Report.pdf

Hermida, Alfred (2010). "Twittering the news. The emergence of ambient journalism". Journalism practice, v. 4, n. 3, pp. 297-308.

https://doi.org/10.1080/17512781003640703

Hermida, Alfred; Thurman, Neil (2008). "A clash of cultures: the integration of user-generated content within professional journalistic frameworks at British newspaper websites". Journalism practice, v. 2, n. 3, pp. 343-356.

https://doi.org/10.1080/17512780802054538

Jacobs, Lawrence R.; Cook, Fay-Lomax; Delli-Carpini, Michael X. (2009). Talking together: Public deliberation and political participation in America. Chicago: University of Chicago Press. ISBN: 9780226389875

Karlsson, Michael; Clerwall, Christer; Nord, Lars (2017). “Do not stand corrected. Transparency and users' attitudes to inaccurate news and corrections in online journalism". Journalism \& mass communication quarterly, v. 94, n. 1, pp. 148167.

https://doi.org/10.1177/1077699016654680

Liu, Jiawei; McLeod, Douglas M. (2019). "Pathways to news commenting and the removal of the comment system on news websites". Journalism, first online.

https://doi.org/10.1177/1464884919849954 
Masip, Pere (2018). "Pluralismo mediático y el futuro del periodismo". Anuario ThinkEPI, v. 12, pp. 203-206. https://doi.org/10.3145/thinkepi.2018.30

Masip, Pere; Ruiz-Caballero, Carlos; Suau, Jaume (2018). Monitoring media pluralism in Europe: application of the media pluralism monitor 2017 in the European Union, Fyrom, Serbia \& Turkey. Country report: Spain. The Centre for Media Pluralism and Media Freedom.

https://doi.org//10.2870/941747

Masip, Pere; Ruiz-Caballero, Carlos; Suau, Jaume (2019). "Active audiences and social discussion on the digital public sphere. Review article". El profesional de la información, v. 28, n. 2, e280204.

https://doi.org//10.3145/epi.2019.mar.04

Mauri-Ríos, Marcel; Ramon-Vegas, Xavier (2015). “Nuevos sistemas de rendición de cuentas de la información periodística. Una exploración del escenario online español”. El profesional de la información, v. 24, n. 4, pp. 380-389.

https://doi.org/10.3145/epi.2015.jul.04

Muñiz, Carlos; Campos-Domínguez, Eva; Saldierna, Alma-Rosa; Dader, José-Luis (2019). “Engagement of politicians and citizens in the cyber campaign on Facebook: A comparative analysis between Mexico and Spain". Contemporary social science, v. 14, n. 1, pp. 102-113.

https://doi.org/10.1080/21582041.2017.1367832

Nieborg, David B.; Poell, Thomas (2018). "The platformization of cultural production: Theorizing the contingent cultural commodity". New media \& society, v. 20, n. 11, pp. 4275-4292.

https://doi.org/10.1177/1461444818769694

Parcu, Pier-Luigi (2019). "New digital threats to media pluralism in the information age". Competition and regulation in network industries, v. 21, n. 2, pp. 91-109.

https://doi.org/10.1177/1783591719886101

Peek, Lori; Fothergill, Alice (2009). “Using focus groups: Lessons from studying daycare centers, 9/11, and hurricane Katrina". Qualitative research, v. 9, n. 1, pp. 31-59.

https://doi.org/10.1177/1468794108098029

Pérez-Díaz, Pedro-Luis; Zamora-Medina, Rocío; Arroyas-Langa, Enrique (2020). "Between self-regulation and participatory monitoring: comparing digital news media accountability practices in Spain”. Media and communication, v. 8, n. 2, pp. 112-123.

https://doi.org/10.17645/mac.v8i2.2721

Porlezza, Colin (2019). "From participatory culture to participatory fatigue: The problem with the public". Social media + society, v. 5, n. 3 .

https://doi.org/10.1177/2056305119856684

Raeijmaekers, Danielle; Maeseele, Pieter (2015). “Media, pluralism and democracy: what's in a name?”. Media, culture \& society, v. 37, n. 7, pp. 1042-1059.

https://doi.org/10.1177/0163443715591670

Ramon-Vegas, Xavier; Rojas-Torrijos, José-Luis; Billings, Andrew C. (2019). “The present (but not future) ESPN ombudsman: Levying accountability through the inception of the digital age". In: Armfield, Greg G.; McGuire, John; Earnheardt, Adam C. (eds.). ESPN and the changing sports media landscape. New York: Peter lang, pp. 185-199. ISBN:978 1433151736

Rodríguez-Martínez, Ruth; Mauri-Ríos, Marcel; Fedele, Maddalena (2017). “Criticism as an accountability instrument: the opinion of Spanish journalists". Communication \& society, v. 30, n. 1, pp. 57-72.

https://doi.org/10.15581/003.30.1.57-72

Ruiz-Caballero, Carlos; Masip, Pere; Micó, Josep-Lluís; Díaz-Noci, Javier; Domingo, David (2010). “Conversación 2.0. y democracia. Análisis de los comentarios de los lectores en la prensa digital catalana". Communication \& society, v. 23, n. 2, pp. 7-39.

https://revistas.unav.edu/index.php/communication-and-society/article/view/36233

Ruiz-Caballero, Carlos; Micó, Josep-Lluís; Díaz-Noci, Javier; Meso-Ayerdi, Koldo; Masip, Pere (2011). "Public sphere 2.0? The democratic qualities of citizen debates in online newspapers". The international journal of press/politics, v. 16, n. 4, pp. 463-487.

https://doi.org/10.1177/1940161211415849

Santana, Arthur D. (2015). "Incivility dominates online comments on immigration”. Newspaper research journal, v. 36, n. 1, pp. 92-107.

https://doi.org/10.1177/073953291503600107 
Spiller, Ralf; Degen, Matthias; Kronewald, Elke; Guertler, Katherine (2016). "Media watchblogs as an instrument of media accountability: An international survey". Journal of applied journalism \& media studies, v. 5, n. 2, pp. $151-176$. https://doi.org/10.1386/ajms.5.2.151_1

Steiner, Miriam; Magin, Melanie; Stark, Birgit (2019). "Uneasy bedfellows. Comparing the diversity of German public service news on television and on facebook". Digital journalism, v. 7, n. 1, pp. 100-123.

https://doi.org/10.1080/21670811.2017.1412800

Suhay, Elizabeth; Bello-Pardo, Emily; Maurer, Brianna (2018). "The polarizing effects of online partisan criticism: Evidence from two experiments". The international journal of press/politics, v. 23, n. 1, pp. 95-115.

https://doi.org/10.1177/1940161217740697

Torres-da-Silva, Marisa (2012). “Newsroom practices and letters-to-the-editor”. Journalism practice, v. 6, n. 2, pp. 250263.

https://doi.org/10.1080/17512786.2011.629124

Tracy, Sarah J. (2020). Qualitative research methods. Chichester, West Sussex: Wiley-Blackwell. ISBN: 9781405192033

Valcke, Peggy; Sükösd, Miklós; Picard, Robert G. (eds.) (2015). Media pluralism and diversity. Concepts, risks and global trends. Houndmills, Basingstoke: Palgrave Macmillan. ISBN: 9781137273291

Valera-Ordaz, Lidia (2017). “Comparación del valor democrático de las discusiones de Facebook entre perfiles de candidatos políticos españoles a las elecciones generales de 2011". Revista internacional de sociología, v. 75, n. 1, e052. https://doi.org/10.3989/ris.2017.75.1.15.119

Valera-Ordaz, Lidia (2019). "Liberal individualist, communitarian, or deliberative? Analyzing political discussion on Facebook based on three notions of democracy". International journal of communication, v. 13, pp. 1056-1076.

https://ijoc.org/index.php/ijoc/article/view/8309

Wahl-Jorgensen, Karin (2002). "Understanding the conditions for public discourse: Four rules for selecting letters to the editor. Journalism studies, v. 3, n. 1, pp. 69-81.

https://doi.org/10.1080/14616700120107347

Wardle, Claire; Derakhshan, Hossein (2017). Information disorder: Toward an interdisciplinary framework for research and policymaking. Estrasburgo: Consejo de Europa.

https://rm.coe.int/information-disorder-toward-an-interdisciplinary-framework-for-researc/168076277c

Weischenberg, Siegfried; Malik, Maja; Scholl, A. (2006). “Journalismus in Deutschland 2005”. Media perspektiven, n. 7, pp. 346-361.

https://www.ard-werbung.de/media-perspektiven/fachzeitschrift/2006/artikel/journalismus-in-deutschland-2005

Wimmer, Roger D.; Dominick, Joseph R. (2011). Mass media research: an introduction (9a edición). Canadá: Wadsworth, Cengage learning. ISBN: 9781439082744

Zeng, Jing; Burgess, Jean; Bruns, Axel (2019). "Is citizen journalism better than professional journalism for fact-checking rumours in China? How weibo users verified information following the 2015 Tianjin blasts". Global media and China, v. 4, n.1, pp. 13-35.

https://doi.org/10.1177/2059436419834124 